\title{
Oral Antibiotic Use Following Stented Hypospadias Repair: Does It Play a Role in the Prevention of Urinary Tract Infections?
}

\author{
Mohammed Suoub (iD ${ }^{1,}{ }^{*}$ and Fadi Sawaqed (iD) ${ }^{1}$ \\ ${ }^{1}$ Department of Special Surgery, Faculty of Medicine, Mut'ah University, Karak, Jordan \\ "Corresponding author: Department of Special Surgery, Faculty of Medicine, Mut'ah University, Karak, Jordan. Email: dr_suoub@yahoo.com
}

Received 2020 April 11; Revised 2020 May 31; Accepted 2020 July 23.

\begin{abstract}
Background: There is a lack of consensus regarding the role of oral antibiotics following hypospadias repair.

Objectives: The study aimed to evaluate the role of oral antibiotic use following stented Tubularized incised plate urethroplasty (TIPU) in the prevention of bacteriuria and urinary tract infections (UTIs).

Methods: A prospective study was conducted on 40 patients undergoing stented TIPU for coronal hypospadias between January 2014 and December 2016. The average age of the subjects was 13.2 months at the time of surgery, ranging from 11 to 16 months. The patients were divided into two groups. Group A consisted of 20 patients receiving oral antibiotics until urethral catheter removal, whereas group B consisted of 20 patients without any oral antibiotics postoperatively.

Results: The two groups were followed for three years. The urethral catheter was removed eight days postoperatively in the two groups. In group A, the patients received oral antibiotics as long as the catheter was left in situ. Urine samples were collected from the patients and sent for analysis and culture at the time of stent removal and after three weeks. The results showed that 3/20 (15\%) patients from group A had pyuria and bacteriuria, while all of them had negative urine culture results. On the other hand, in group $\mathrm{B}, 8 / 20$ (40\%) patients had pyuria and bacteriuria ( $\mathrm{P}>0.05$ ), and four (20.0\%) patients had positive urine cultures for Escherichia coli $(\mathrm{P}<0.05)$, sensitive to co-trimoxazole. None of the patients in the two groups had febrile UTI.

Conclusions: The use of oral antibiotics for patients following stented TIPU reduces pyuria and significantly decreases positive urine culture results and the risk of UTI after surgery.
\end{abstract}

Keywords: Hypospadias, Urinary Tract Infections, Antibiotic, Stent

\section{Background}

Tubularized incised plate urethroplasty (TIPU) is currently applied as the best surgical approach for distal shaft hypospadias (1-3). Evidence shows that the use of a ureteral stent for hypospadias repair can reduce postoperative complications, namely, fistula rate and meatal stenosis (4-6). Recently, the use of oral prophylactic antibiotics has been challenging following distal hypospadias repair with stenting (7). Some previous studies have suggested that prophylactic antimicrobial treatment may prevent prolonged indwelling catheterization in patients with urinary tract infections (UTIs) (8). On the other hand, some studies have suggested that oral prophylactic antibiotics may not be needed in stented hypospadias repair (9).

\section{Objectives}

This study aimed to assess the efficacy of oral antibiotic administration in preventing pyuria, bacteriuria, and pos- itive urine culture results following stented TIPU

\section{Methods}

A prospective study was conducted after obtaining approval from the Institutional Ethics Committee of the Faculty of Medicine (Mu'tah University, Mu'tah, Jordan) between January 2014 and December 2017. The study included 40 patients undergoing distal hypospadias repair. The inclusion criteria were as follows: (1) a diagnosis of distal hypospadias; (2) age range of 11 - 16 months; and (3) living near the hospital for a better follow-up. Written informed consent was obtained from the parents/guardians of the patients included in the study.

The patients were randomly divided into two groups. Group A consisted of 20 patients who underwent TIPU and received oral antibiotics postoperatively as long as the ureteral stent was left in situ. Group B included 20 consecutive patients (matched with group A for age and time of 
surgery) who underwent TIPU and received no oral antibiotics postoperatively.

All patients received one intravenous dose of a thirdgeneration cephalosporin (100 $\mathrm{mg} / \mathrm{kg}$ BW of ceftriaxone) for anesthesia induction. All surgeries were performed by a single surgeon. A two-layer de-epithelialized dartos flap was used as a barrier between the neourethra and skin to reduce the fistula rate $(10,11)$. For all patients, the urinary diversion was performed using a urethral stent (7 Fr soft feeding tube), fixed to the glans with 5/0 Prolene sutures for eight days. The postoperative dressing was done with a local antibiotic ointment (fusidic acid) using a Tegaderm bandage, followed by a Coban compression dressing. The method of dressing was the same for all patients.

To avoid fecal soiling in the ureteral stent, the stent was kept short and unfolded between the two diapers and fixed with a tape close to the scrotum neck. All patients had an uneventful procedure and hospital stay and were discharged on the day of surgery. Before discharging the patients, their families were provided with instructions on how to handle the urethral stent, clean the genital area, and avoid fecal soiling on the stent and penis.

On the day of surgery, group A started receiving the syrup form of co-trimoxazole as an antibiotic, consisting of sulfamethoxazole (40 mg) and trimethoprim ( $8 \mathrm{mg} / \mathrm{kg}$ BW), in two separate doses every 12 hours to prevent Gramnegative bacilli. On the other hand, patients in group $B$ were discharged from the hospital without any oral antibiotics. On the following day, the Coban compression dressing was removed at home by the parents, and the rest of the dressing was kept until the first visit to the clinic.

In the first follow-up, which was eight days after the surgery and before ureteral stent and Tegaderm removal, the stent was clamped for 30 minutes, and its tip was cleaned with povidone-iodine as an antiseptic to prevent contamination. Next, it was washed with normal saline, and the first $10 \mathrm{cc}$ of the urine sample was discarded. The rest of the urine sample was collected in a sterile container for urinalysis and culture.

\section{Results}

The mean age of the patients was 13.2 months (range: 11 - 16 months) at the time of surgery. Three (15\%) patients from group A had positive pyuria $(\mathrm{WBC}+\mathrm{VE})($ Table 1$)$, while all of them had negative urine culture results (culture-VE). In group B, eight (40\%) patients had positive pyuria (Table 1 ), and four (20\%) patients had positive urine culture results for E. coli $(\mathrm{CX}+\mathrm{VE})$ (Table 2).

A chi-square test was performed in IBM SPSS version 22 to determine if the postoperative use of oral antibiotics was associated with the results of urinalysis for positive

\begin{tabular}{cccc}
\hline Table 1. The Urinalysis Results ${ }^{\mathrm{a}}$ & \multicolumn{2}{c}{} & \\
\hline & \multicolumn{2}{c}{ WBC } & Total \\
\cline { 2 - 3 } & \multicolumn{2}{c}{$-\mathbf{V E} \quad+\mathbf{V E}$} & \\
\hline Group & & & \\
\hline A & & & 20 \\
\hline Count & 17 & 3 & 100.0 \\
\hline Within group, \% & 85.0 & 15.0 & \\
\hline B & & & 20 \\
\hline Count & 12 & 8 & 100.0 \\
\hline Within group, \% & 60.0 & 40.0 & \\
\hline Total & & & 40 \\
\hline Count & 29 & 11 & 100.0 \\
\hline Within group, \% & 72.5 & 27.5 & \\
\hline
\end{tabular}

${ }^{\text {a }}$ Pearson's chi-square $=0.077$.

\begin{tabular}{|c|c|c|c|}
\hline & \multicolumn{2}{|c|}{ Culture } & \multirow{2}{*}{ Total } \\
\hline & $-V E$ & $+\mathbf{V E}$ & \\
\hline \multicolumn{4}{|l|}{ Group } \\
\hline \multicolumn{4}{|l|}{ A } \\
\hline Count & 20 & 0 & 20 \\
\hline Within group, \% & 100.0 & 0.0 & 100.0 \\
\hline \multicolumn{4}{|l|}{ B } \\
\hline Count & 16 & 4 & 20 \\
\hline Within group, \% & 80.0 & 20.0 & 100.0 \\
\hline \multicolumn{4}{|l|}{ Total } \\
\hline Count & 36 & 4 & 40 \\
\hline Within group, \% & 90.0 & 10.0 & 100.0 \\
\hline
\end{tabular}

${ }^{\mathrm{a}}$ Pearson's chi-square $=0.035$.

WBC and the results of urine culture for positive bacterial growth. The proportion of subjects with positive WBC was not significantly different in terms of oral antibiotic use $\left(\chi^{2}(1, \mathrm{n}=20)=3.135 ; \mathrm{P}>0.05\right)$. There was a significant relationship between oral antibiotic use and culture results. In other words, patients who did not receive oral antibiotics were more likely to have positive urine culture results than those who received oral antibiotics $\left(\chi^{2}(1, \mathrm{n}=20)=4.444 ; \mathrm{P}\right.$ $<0.05)$.

The patients with positive urine cultures $(n=4)$ received oral antibiotics, according to the antibiotic sensitivity results. None of the patients in the two groups with positive pyuria and bacteriuria or positive urine culture results had a fever or symptomatic infection.

The second follow-up was scheduled for all patients 
three weeks after the stent removal, and urine samples were collected for analysis and culture, using pediatric urine bags. The results were negative for pyuria and bacteriuria, and the urine culture was negative, as well.

\section{Discussion}

In this study, we investigated the benefits of oral antibiotic use after hypospadias repair. The results showed the reductions in pyuria and positive urine culture results, which might, in turn, prevent serious ascending UTIs, especially if the anomaly is accompanied by other pathologies in the urinary tract, such as vesicoureteral reflux (VUR). In this regard, Kim et al. (12) studied the clinical course of VUR in patients with hypospadias. They reported a higher prevalence of VUR in these children than in healthy children.

Tubularized incised plate urethroplasty, which was first described by Snodgrass in $1994(13,14)$, is the most acceptable surgical approach for distal penile hypospadias to reduce known surgical complications, such as fistula formation and meatal stenosis. The use of stents following hypospadias repair is recommended for an average of seven days. This suggestion was supported by Buson et al. (5), who found that stents were advantageous following repair surgeries, with a higher complication rate in stentless cases than in stented cases (18.9\% vs. $4.6 \%)$; therefore, they recommended the use of stents during hypospadias repair. Overall, most previous studies advocate that patients should receive oral antibiotics to prevent UTI as a major concern after temporary postoperative urinary stent drainage $(7,8)$.

On the other hand, Smith et al. (15), in a study on $224 \mathrm{pa}-$ tients, reported that the use of preoperative antibiotics did not reduce the rate of infection at the surgical site following hypospadias repair. Moreover, Kanaroglou et al. (9), in a retrospective study at SickKids Hospital (Canada) on newborns without oral antibiotics after hypospadias surgery, reported no increase in the incidence of symptomatic UTIs or complications. The comparison of our results with the study by Kanaroglou et al. (9) indicates that no antibiotic use following hypospadias repair can cause symptomatic infections or adverse consequences.

The main limitation of this study was the small number of patients, leading to low statistical power. We concluded that the use of oral antibiotics following stented TIPU could significantly reduce bacteriuria, pyuria, and positive urine culture results, which might, in turn, prevent upper UTIs.

\subsection{Conclusions}

Although there is a lack of consensus regarding the role of oral antibiotics following hypospadias repair, the present results showed that oral antibiotic use after stented TIPU could reduce pyuria; it also significantly decreased the positive urine culture results and the risk of UTI after surgery.

\section{Acknowledgments}

We extend our gratitude to all personnel who contributed to this study.

\section{Footnotes}

Authors' Contribution: Study concept and design: MS. Analysis and interpretation of data: MS and FS. Drafting of the manuscript: MS. Critical revision of the manuscript for important intellectual content: FS. Statistical analysis: MS and FS. Administrative, technical, and material support: MS

Conflict of Interests: We declare that there is no conflict of interest regarding the publication of this paper.

Ethical Approval: The Ethics Committee of Mu'tah Faculty of Medicine approved this study (ref. no.: 201456).

Funding/Support: This study was not funded by any sources.

Informed Consent: Written informed consent forms were obtained from the parents/guardians of the patients at the time of scheduling the surgery.

\section{References}

1. Snodgrass W, Koyle M, Manzoni G, Hurwitz R, Caldamone A, Ehrlich R. Tubularized incised plate hypospadias repair: Results of a multicenter experience. J Urol. 1996;156(2 Pt 2):839-41. doi: 10.1097/00005392199608001-00082. [PubMed: 8683797].

2. Manzoni G, Bracka A, Palminteri E, Marrocco G. Hypospadias surgery: When, what and by whom? BJU Int. 2004;94(8):1188-95. doi: 10.1046/j.1464-410x.2004.05128.x. [PubMed: 15613162].

3. Braga LH, Lorenzo AJ, Salle JL. Tubularized incised plate urethroplasty for distal hypospadias: A literature review. Indian J Urol. 2008;24(2):219-25. doi: 10.4103/0970-1591.40619. [PubMed: 19468401]. [PubMed Central: PMC2684277].

4. Chang PC, Yeh ML, Chao CC, Chang CJ. Use of double pigtail stent in hypospadias surgery. Asian J Surg. 2011;34(1):28-31. doi: 10.1016/S10159584(11)60015-8. [PubMed: 21515210].

5. Buson H, Smiley D, Reinberg Y, Gonzalez R. Distal hypospadias repair without stents: Is it better? J Urol. 1994;151(4):1059-60. doi: 10.1016/s0022-5347(17)35180-7. [PubMed: 8126792].

6. Almodhen F, Alzahrani A, Jednak R, Capolicchio JP, El Sherbiny MT. Nonstented tubularized incised plate urethroplasty with Y-toI spongioplasty in non-toilet trained children. Can Urol Assoc J. 2008;2(2):110-4. doi: 10.5489/cuaj.484. [PubMed: 18542743]. [PubMed Central: PMC2422900]. 
7. Canon S, Marquette MK, Crane A, Patel A, Zamilpa I, Bai S. Prophylactic antibiotics after stented, distal hypospadias repair: Randomized pilot study. Glob Pediatr Health. 2018;5:2333794X18770074. doi: 10.1177/2333794X18770074. [PubMed: 29761139]. [PubMed Central: PMC5946338].

8. Shohet I, Alagam M, Shafir R, Tsur H, Cohen B. Postoperative catheterization and prophylactic antimicrobials in children with hypospadias. Urology. 1983;22(4):391-3. doi: 10.1016/0090-4295(83)90417-x. [PubMed: 6636394].

9. Kanaroglou N, Wehbi E, Alotay A, Bagli DJ, Koyle MA, Lorenzo AJ, et al. Is there a role for prophylactic antibiotics after stented hypospadias repair? J Urol. 2013;190(4 Suppl):1535-9. doi: 10.1016/j.juro.2013.02.015. [PubMed: 23416639].

10. Kamal BA. Double dartos flaps in tubularized incised plate hypospadias repair. Urology. 2005;66(5):1095-8. doi: 10.1016/j.urology.2005.05.020. [PubMed: 16286134].

11. Bakan V, Yildiz A. Dorsal double-layer dartos flap for preventing fistu- lae formation in the Snodgrass technique. Urol Int. 2007;78(3):241-4 doi: 10.1159/000099345. [PubMed: 17406134].

12. Kim KH, Lee HY, Im YJ, Jung HJ, Hong $\mathrm{CH}$, Han SW. Clinical course of vesicoureteral reflux in patients with hypospadias. Int J Urol. 2011;18(7):521-4. doi: 10.1111/j.1442-2042.2011.02777.x. [PubMed: 21605171].

13. Snodgrass WT, Bush N, Cost N. Tubularized incised plate hypospadias repair for distal hypospadias. J Pediatr Urol. 2010;6(4):408-13. doi: 10.1016/j.jpurol.2009.09.010. [PubMed: 19837000].

14. Cheng EY, Vemulapalli SN, Kropp BP, Pope J, Furness P3, Kaplan WE, et al. Snodgrass hypospadias repair with vascularized dartos flap: The perfect repair for virgin cases of hypospadias? JUrol. 2002;168(4 Pt 2):1723-6. discussion 1726. doi: 10.1097/01.ju.0000026940.33540.31. [PubMed: 12352344].

15. Smith J, Patel A, Zamilpa I, Bai S, Alliston J, Canon S. Analysis of preoperative antibiotic prophylaxis in stented, distal hypospadias repair. Can J Urol. 2017;24(2):8765-9. [PubMed: 28436366]. 